\title{
Redes sociales como espacio de aprendizaje: codiseño de estrategias de enseñanza para abordar la Pandemia Social media as a learning space: co-design of teaching strategies to address the Pandemic
}

\author{
Czerwonogora, A., Doninalli, M., Rodríguez Enríquez, C. \\ aczerwonogora@fenf.edu.uy, fdonmar@hotmail.com, carolinacabocla@gmail.com \\ Unidad de Enseñanza Virtual y de Aprendizaje \\ Facultad de Enfermería, Universidad de la República \\ Uruguay
}

\begin{abstract}
Resumen- El presente trabajo presenta una investigación de métodos mixtos, centrada en el análisis de dos ediciones de una Unidad Curricular de la carrera de Licenciatura en Enfermería, Universidad de la República, Uruguay, periodos 2020 y 2021. La Unidad fue codiseñada en el marco del contexto sanitario mundial, enfocada en la educación como un derecho humano a mantener. El escenario de pandemia ha volcado a las personas en las redes sociales como espacio de interacción social. Estas se convirtieron en ambiente de aprendizaje y cooperación tanto para los estudiantes universitarios como para la comunidad. En el estudio se destacan las habilidades específicas de la profesión enfermera como su esencia profesional: las habilidades blandas y el cuidado. Se entiende que las y los estudiantes evidencian solvencia para desarrollar actividades colaborativas enfocadas en su rol profesional, en un contexto de crisis. Los resultados obtenidos confirman las posibilidades de aplicación del aprendizaje colaborativo mediado como enfoque para evaluar actividades de enseñanza y aprendizaje complejas en entornos virtuales en el ámbito universitario.
\end{abstract}

Palabras clave: aprendizaje colaborativo mediado; educación en Enfermería; redes sociales; cuidado.

Abstract- The paper presents a mixed methods research, focused on the analysis of two editions of an undergraduate course of Nursing Degree from Universidad de la República, Uruguay, periods 2020 and 2021. The course was co-designed within the global health crisis context, focused on education as a human right to maintain. The pandemic scenario has turned people into social media as a place for social interaction. They became an environment for learning and cooperation for both college students and the community. The study highlights the specific skills of the nursing profession as its professional essence: soft skills and care. The students show solvency to develop collaborative activities focused on their professional role, in a context of crisis. The results obtained confirm the possibilities of applying computer supported collaborative learning as a valid approach to evaluate complex teaching and learning activities in virtual environments in higher education.

Keywords: Computer Supported Collaborative Learning; Nursing education; social media; care.

\section{INTRODUCCIÓN}

La apresurada evolución de la conectividad a partir de una necesidad de conexión, que condujo a los usuarios a acercarse a las redes sociales, resultó moldeadora de la socialidad en línea. En este sentido, el desarrollo de las tecnologías de la información y la comunicación, cuyo progreso en los últimos dos siglos se ha generado como parte de las tendencias culturales, aportó a esta evolución al permitir que las actividades cotidianas se trasladaran a los espacios en línea (Van Dijck, 2016).

Las redes sociales han adquirido importancia como plataformas capaces de colaborar con la disciplina de Enfermería para la adquisición por parte de los estudiantes de una mayor comprensión relativa a la comunicación, profesionalismo, políticas de salud y ética profesional. Las enfermeras constituyen importantes agentes de conocimiento dentro de los sistemas de salud, como disciplina que centra su desarrollo en el cuidado tanto con pacientes, sus familias y la comunidad. Por este motivo la educación en Enfermería explora formas para integrar la aplicación de estos medios al currículum (Schmitt, Sims-Giddens, y Booth, 2012). En los pregraduados, el desconocimiento de su uso adecuado puede impactar en forma negativa en sus posibilidades laborales (Edge, 2017). En todos los casos de uso, a nivel personal, en el ámbito profesional, así como en el marco institucional como promotor de cultura organizacional, puede beneficiarse a través de una práctica reflexiva (Jackson, Fraser y Hash, 2014).

En esta línea, Rodríguez y Barbieri (2014) manifiestan la necesidad de incorporar a la discusión el uso de las redes sociales para compartir en diversos ámbitos de actuación profesional el sentir de Enfermería. La difusión de actividades e información institucional a través de una red social amplía el alcance al permitir llegar a un mayor público objetivo y contribuye a la construcción social del rol de Enfermería. Si bien las autoras se refieren en su estudio a la red social Facebook, no resulta diferente ante el uso de otras redes sociales, aunque sus lógicas de publicación difieran.

Por su parte, Rodríguez y Doninalli (2021) mencionan que es la capacidad multiplicadora de estas redes, en tanto fortaleza, 
lo que beneficiaría la generación de prácticas educativas fuera de los espacios y tiempos formales.

\section{CONTEXTO}

"Uso adecuado de redes sociales: una responsabilidad ciudadana", forma parte de la oferta de materias optativas para estudiantes de Fenf que cursan el mencionado plan de estudios y como asignatura electiva para otros servicios del área de ciencias de la salud de la Udelar. Fue inicialmente planificada para desarrollarse en modalidad semipresencial. La asignatura incluía encuentros en espacios abiertos cercanos al edificio de la Fenf, fuera de los ámbitos formales habituales, e interacción en las redes sociales a trabajar, extendiendo el aula al incorporar otros ambientes a los contextos de enseñanza y de aprendizaje. A partir de la situación de emergencia sanitaria que planteó, entre tantos, un desafío al sistema educativo, se requirió una readaptación de las propuestas educativas a nivel institucional, con el fin de que los estudiantes avanzaran con aquellas asignaturas que pudieran codiseñarse para adecuarse a una modalidad en línea. Tal fue el caso de este curso, que incorporó un aula en el Entorno Virtual de Aprendizaje (EVA), plataforma designada por la Udelar para dar soporte a las clases en línea y el trabajo grupal (Rodríguez y Doninalli, 2021). Hasta el momento se realizaron dos ediciones, dictadas en junio de 2020 y marzo de 2021, ambas en el contexto de pandemia.

Como expresa Gros (2019), el empleo de métodos de codiseño deriva de la disciplina del diseño participativo. En el ámbito educativo, la autora sostiene que:

“(...) lo más habitual ha sido implicar a los docentes en el codiseño de acciones, métodos y productos de apoyo al aprendizaje. Este tipo de enfoque ha permitido desarrollar innovaciones enraizadas en los contextos reales de enseñanza y aprendizaje, facilitando la apropiación de nuevas herramientas y estrategias y promoviendo la transformación profunda de las prácticas educativas. En estas situaciones, las necesidades y problemas se plantean como cuestiones abiertas y complejas que requieren de un abordaje global. De este modo, se invita a los participantes a poner en juego diversidad de perspectivas y de estrategias para desarrollar distintas soluciones posibles." (p.8) En las aulas virtuales de plataformas como EVA, las discusiones asincrónicas en los foros brindan la oportunidad de interactuar entre pares y constituyen una de las herramientas más importantes para el aprendizaje (Kent, Laslo y Rafaeli, 2016) entendido como construcción social (Vigotsky, 1978). En este sentido, el aprendizaje colaborativo mediado estudia la participación en estos espacios como una de las variables fundamentales asociadas al aprendizaje en línea (Chávez, Montaño, Barrera, Sánchez y Faure, 2021 y referencias citadas allí).

El objetivo de esta investigación es analizar y comparar la participación de las y los estudiantes en el foro de trabajo colaborativo en EVA de las dos ediciones de la asignatura "Uso de redes sociales: una responsabilidad ciudadana" realizadas en el contexto de emergencia sanitaria.

\section{A. Propuesta de trabajo colaborativo en el curso}

El trabajo colaborativo se propuso de forma transversal en el curso, con diferentes niveles, diversos espacios y momentos. El objetivo era diseñar, colaborativamente y en línea, materiales didácticos a difundir por redes sociales. Se inició con la conformación de subgrupos según preferencia de los estudiantes por una red social de las tres ofertadas (Facebook, Instagram y Twitter) con un docente como referente. La actividad comenzó en un foro de debate e intercambio para cada subgrupo en el EVA, donde debían acordar la temática a abordar, entender que generaba un mensaje de cuidado, enfocado en la realidad, en un contexto de pandemia. Cabe aclarar que inicialmente la temática a abordar sería sobre el cuidado, esencia de la disciplina enfermera y en un mes donde se conmemora. El contexto redireccionó el abordaje del cuidado en tiempos de pandemia. La evaluación estudiantil del curso y continuar en contexto de pandemia para la segunda edición, determinó este aspecto del codiseño, donde se mantuvo la elección de las temáticas por ellos por su alta valoración e impacto.

Luego de seleccionada la temática debían diseñar cinco materiales (a ser publicados uno por día hábil en el marco de una semana común). El diseño de los materiales y mensajes debía seguir las lógicas de cada red social, considerando las orientaciones docentes y tener licenciamiento abierto. El tiempo total de trabajo de los equipos fue de dos semanas (producción y publicación).

La producción de contenidos y posterior interacción de los estudiantes y comunidad con los contenidos en las redes sociales, están vinculados a la profesión y competencias de los Licenciados en Enfermería: impartir cuidados destacando las habilidades blandas propias de la experticia profesional en el proceso, a las que se suman las tecnologías de la información y comunicación, particularmente las redes sociales. Este fue otro espacio de trabajo colaborativo al compartir los contenidos, comentarlos, difundirlos, propagarlos.

\section{DESCRIPCIÓN}

Se propone una investigación de métodos mixtos (Creswell y Plano, 2018), enfocada en el análisis de participación (Czerwonogora, 2017) de ambas ediciones de la asignatura.

\section{B. Análisis de participación}

El análisis de participación se centró en el foro de trabajo colaborativo en equipos. La participación de los estudiantes en este foro se definió como el promedio de mensajes enviados en cada hilo de foro (Schrire, 2006) y se consideraron solo los estudiantes activos. El análisis cuantitativo se realizó con apoyo de una planilla de cálculo electrónica. En los hilos de foro estudiados pertenecientes a cada equipo se contabilizaron los mensajes y se calcularon los promedios correspondientes. Todos los conteos fueron revisados con las herramientas de registro disponibles en la plataforma educativa (summary report de Moodle para cada tema del foro).

\section{Análisis de contenido}

La naturaleza de la comunicación referida a los contenidos de los mensajes del foro se clasificó en tres categorías: asociada 
a la tarea, de regulación y social (Lockhorst, 2004; 2010; tabla 1). En cada categoría se consideró la proporción de mensajes correspondientes y el contenido predominante en cada mensaje. Durante el análisis se revisaron y corrigieron las categorías para ajustarlas a las observadas en los textos (Gibbs, 2012). No se codificaron las intervenciones docentes ya que el estudio está centrado en la participación estudiantil.

Tabla 1

Tipo de comunicación según el contenido de mensaje enviado al foro

\begin{tabular}{|l|l|}
\hline \multicolumn{1}{|c|}{$\begin{array}{c}\text { Tipo de } \\
\text { comunicación }\end{array}$} & \multicolumn{1}{|c|}{ Descripción } \\
\hline $\begin{array}{l}\text { Contenido } \\
\text { relacionado con } \\
\text { la tarea }\end{array}$ & $\begin{array}{l}\text { Mensajes referidos a actividades y } \\
\text { acciones vinculadas a la tarea asignada. }\end{array}$ \\
\hline De regulación & $\begin{array}{l}\text { Mensajes referidos a observaciones sobre } \\
\text { la planificación de la tarea, roles en la } \\
\text { tarea, evaluaciones sobre el proceso del } \\
\text { grupo y el trabajo realizado, supervisión } \\
\text { general de la tarea, cuestiones de } \\
\text { organización, preguntas sobre la tarea. }\end{array}$ \\
\hline Social & $\begin{array}{l}\text { Mensajes de comunicación que si bien se } \\
\text { refieren a la tarea, se enfocan en la } \\
\text { apreciación de las contribuciones de los } \\
\text { participantes, incluyendo interacciones; } \\
\text { saludos. }\end{array}$ \\
\hline
\end{tabular}

\section{Resultados}

En ambas ediciones del curso se completaron las plazas de inscripciones: 88 y 85 estudiantes en 2021 y 2020, respectivamente. A los efectos del estudio se consideraron aquellos estudiantes activos en el foro, pertenecientes a los equipos de trabajo en cada red social. El número de estudiantes participantes en cada equipo fue similar en las dos ediciones (Tabla 2). En ambos casos se constató mayor interés en integrar el equipo correspondiente a la red Instagram (fue el cupo que se completó primero), seguido por el de Facebook. El equipo de Twitter fue el que tuvo menor convocatoria, y en algunos casos fue seleccionado por descarte. Estas observaciones concuerdan con la encuesta realizada en el marco del curso ( $N=70$ en 2021; $\mathrm{N}=69$ en 2020), cuyos resultados indicaron que solo una minoría de estudiantes señaló a Twitter como su red social favorita $(11,4 \%$ en $2021 ; 8,7 \%$ en 2020$)$. Por otra parte, la red social preferida fue Instagram (55,7\% en $2021,44,9 \%$ en 2020$)$ y Facebook (10\% en 2021, 18,8\% en 2020) ocupó el tercer lugar en la segunda edición. Asimismo, fueron escogidas otras redes sociales como favoritas $(21,4 \%$ en $2021 ; 26 \%$ en 2020$)$ y en ambas ediciones un participante marcó la no preferencia por ninguna red.

\section{Análisis de participación}

En la tabla 2 se presentan los resultados generales comparados correspondientes a cada red social. Se analizaron en total 363 mensajes de 2021 y 265 mensajes de 2020.

Tabla 2

Mensajes enviados en el foro colaborativo en cada equipo

\begin{tabular}{|l|c|c|c|c|c|c|}
\hline Red social & \multicolumn{2}{|c|}{ Instagram } & \multicolumn{2}{c|}{ Facebook } & \multicolumn{2}{c|}{ Twitter } \\
\hline Edición & 2021 & 2020 & 2021 & 2020 & 2021 & 2020 \\
\hline $\begin{array}{l}\text { Estudiantes } \\
\text { activos }\end{array}$ & 28 & 28 & 27 & 24 & 13 & 13 \\
\hline $\begin{array}{l}\text { Cantidad de } \\
\text { mensajes }\end{array}$ & 123 & 127 & 146 & 81 & 94 & 57 \\
\hline $\begin{array}{l}\text { Promedio de } \\
\text { mensajes }\end{array}$ & 4 & 5 & 5 & 3 & 7 & 4 \\
\hline
\end{tabular}

En la figura 1 se comparan los mensajes enviados por los equipos de trabajo colaborativo en los foros correspondientes a cada red social, para cada edición de la asignatura. Se constató que el porcentaje de mensajes fue similar en ambas ediciones para el caso de Twitter (25,9\% en 2021 y $21,5 \%$ en 2020$)$, pero bastante superior para Facebook en la edición $2021(40,2 \%)$ y para Instagram en $2020(47,9 \%)$.

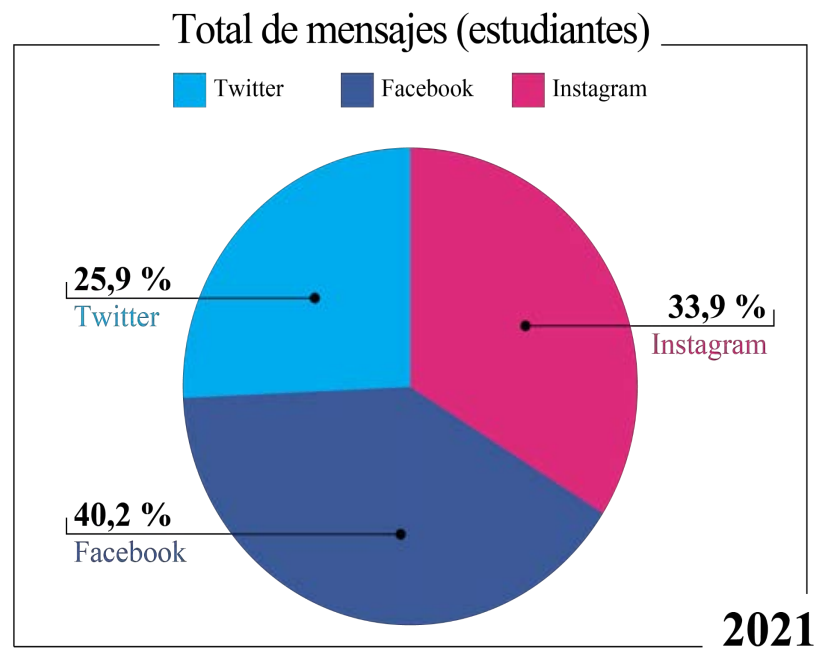




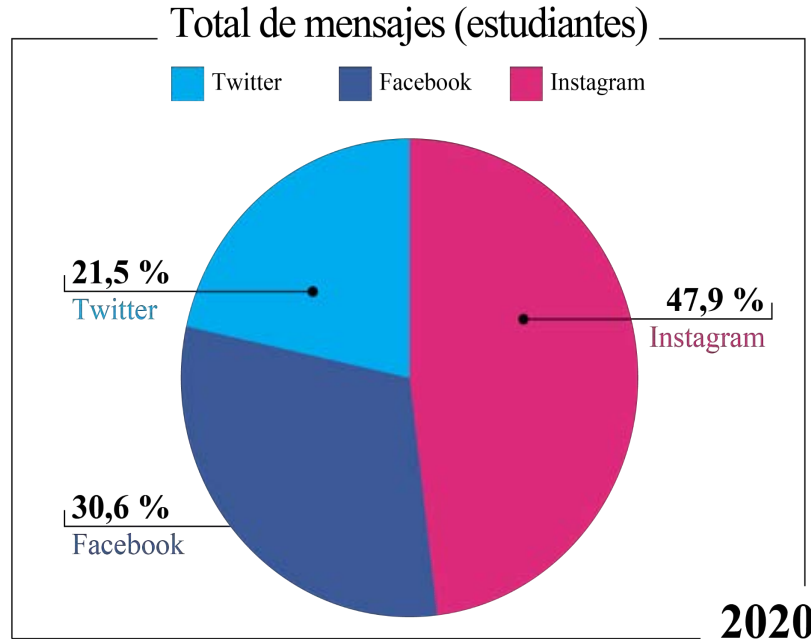

Figura 1 Mensajes enviados en los foros de trabajo colaborativo de los equipos según la red social seleccionada para las ediciones $2021(\mathrm{~N}=363)$ y $2020(\mathrm{~N}=265)$ del curso.

\section{E. Análisis de contenido}

En la figura 2 se presentan los resultados comparados del análisis de los tipos de comunicación observados de acuerdo al contenido de los mensajes enviados por las y los estudiantes en los foros de trabajo colaborativo de los equipos correspondientes a cada red social.

En los tres foros predominaron los mensajes referidos al contenido (cuidado y pandemia) de la tarea a desarrollar y acciones asociadas con la misma, para las dos ediciones del curso. Sin embargo, se constató un mayor porcentaje de estos mensajes en 2020, para los equipos de Instagram $(59,1 \%)$ y Facebook (44,4\%). Nuevamente, los mensajes de contenido de los grupos de Twitter presentaron porcentajes similares $(62,8 \%$ y $59,6 \%$ para 2021 y 2020 , respectivamente). En los equipos de ambas ediciones se enfatizó en el rol de la enfermería y del personal de la salud en la pandemia, así como en la prevención, destacando la higiene de manos como un aspecto central en el cuidado. Las temáticas seleccionadas estuvieron relacionadas con el momento que estaban viviendo las y los estudiantes de cada edición, no solo desde lo personal sino a nivel de la situación general del país con relación a la pandemia.

Un aspecto a señalar fue que los equipos de trabajo de la edición 2020 profundizaron más en la búsqueda de materiales sobre la temática a desarrollar que sus pares de la siguiente edición del curso; no solamente buscaron páginas web sobre la temática, también compartieron artículos científicos. Estos recursos fueron luego utilizados como fuente para la producción. Esta edición del curso fue muy próxima a la declaración de emergencia sanitaria en Uruguay (13 de marzo de 2020). Por este motivo, los equipos abordaron aspectos que resultaban más preocupantes al inicio local de la emergencia, como el uso correcto de las mascarillas y mitos y verdades sobre el COVID-19. También, asociado al contexto de aislamiento (cuarentena, aunque no obligatoria) que acompañó la primera etapa en el país, se interesaron por la salud mental, en particular de los menores de 30 años, así como por el aumento de las $\underline{\text { situaciones de violencia y en especial de la relacionada con el }}$ género.

En la edición 2021 nuevamente fue protagonista la temática de la salud mental, más enfocada en el cuidado en general. La importancia y el incentivo hacia la vacunación fue muy importante en los equipos de este año, en sintonía con la situación general del Uruguay que inició la campaña de vacunación el 27 de febrero; también elaboraron publicaciones referidas a verdades y mitos de las vacunas.

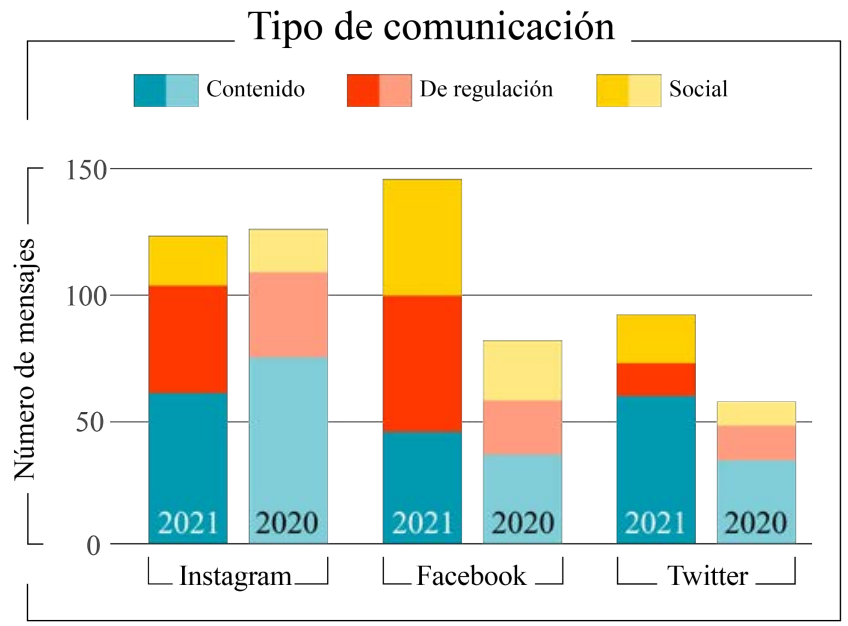

Figura 2 Análisis del tipo de comunicación según el contenido de los mensajes enviados a los foros de cada red social para las ediciones 2021 y 2020 del curso.

En líneas generales, en segundo lugar los mensajes se refirieron a la regulación de la tarea planteada. En 2021 se observaron porcentajes similares en Facebook (37,5\%) e Instagram (35,0\%), superiores a los de 2020 en ambos equipos (27,2\% y $26,8 \%$, respectivamente). En el caso de los equipos que trabajaron en Twitter, se dio una situación inversa: el mayor porcentaje de mensajes se constató en 2020 (24,6\%), frente a los enviados en 2021 (14,9\%). En estos mensajes los participantes de los equipos se organizaron y planificaron a partir de las ideas y contenidos propuestos, sugirieron aspectos a mejorar en las publicaciones a realizar y también intercambiaron sobre aspectos técnicos, referidos a las aplicaciones y programas utilizados para trabajar. Compartieron comentarios sobre el diseño y colores para sus publicaciones, tomaron nota de las recomendaciones del equipo docente y continuaron la elaboración a partir de todas las sugerencias, siempre con ánimo y tono constructivo.

En todos los equipos de trabajo de ambas ediciones una parte de la comunicación se centró en mensajes de contenido social. Estos mensajes mostraron mayores porcentajes en los equipos de trabajo en Facebook (31,5\% en 2021 y 28,4\% en 2020); fueron similares y menores en los equipos de Instagram $(15,4 \%$ en 2021 y $14,2 \%$ en 2020).

En la comunicación de base textual, la presencia social resulta un desafío, y a la vez es crucial para la colaboración. 
Involucra la afectividad, facilitadora del diálogo, y la comunicación abierta, que promueve la participación; ambas tienen efecto en la cohesión del grupo (Garrison y Anderson, 2005). Las y los estudiantes se manifestaron de acuerdo con las ideas de sus compañeros y apreciaron las producciones de sus pares y los logros alcanzados en el grupo, reconociendo el valor del trabajo de su equipo.

En la figura 3 se observan algunos de los resultados de las producciones de los equipos, compartidas desde las cuentas oficiales de la Fenf.

La mediación tecnológica de las computadoras y otros dispositivos digitales ofrece oportunidades que potencian las interacciones (Cress, Stahl, Ludvigsen y Law, 2015). Consideramos que los intercambios en los foros de los equipos son una muestra de esa posibilidad de interacción. Compartimos la idea de que la colaboración en estos espacios es mucho más que solo comunicación entre individuos que comparten información, intercambian ideas o coordinan actividades, sino que crean en conjunto algo nuevo: nuevo conocimiento o comprensión que los participantes no poseían previamente. Scardamalia y Bereiter (2006) visualizan que quienes aprenden pueden construir conocimiento colectivamente al responsabilizarse por mejorar su comprensión sobre problemas auténticos. El aprendizaje colaborativo constituye una estrategia que mejora la adquisición y retención del conocimiento, a la vez que ayuda a las y los estudiantes a enfrentarse a situaciones de resolución de problemas, expresar ideas y desarrollar el pensamiento crítico (Muñoz-Repiso, Gómez-Pablos y García, 2014). En este sentido, el trabajo colaborativo de los equipos se constituyó en un espacio de reflexión, debate, interacción, acuerdos, donde aprender sobre las redes sociales y el rol profesional en estas; un espacio para adquirir competencias generales relacionadas con las tecnologías de la información y la comunicación, enfocado en el rol profesional. Este rol requiere que desde el proceso educativo se generen capacidades de producción de contenidos pensados y destinados a la sociedad, brindando cuidados y visibilizando las especificidades de la profesión y su quehacer.

La pandemia ha destacado las habilidades (blandas) y competencias esenciales requeridas para estar preparado para enfrentar una situación de crisis, que más allá de la competencia digital involucra la evaluación crítica y la capacidad de analizar la información y su veracidad (Bozkurt et al. 2020). Las producciones colaborativas de los equipos también son un ejemplo de ese aprendizaje. Para los profesionales de Enfermería lo innovador es el medio, no el cuidado ni las habilidades blandas, específicas de esta disciplina.

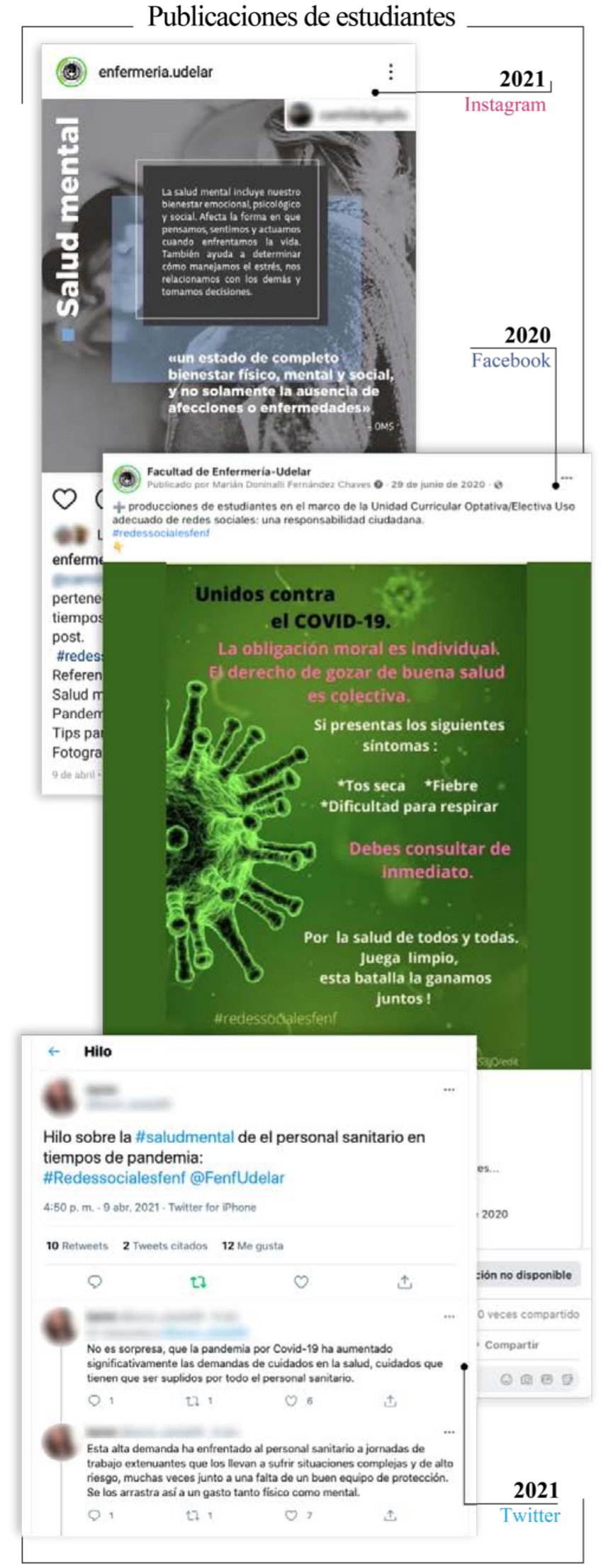


Figura 3 Imágenes de publicaciones producidas por las y los estudiantes y compartidas en sus cuentas en las redes sociales Instagram, Facebook y Twitter.

\section{CONCLUSIONES}

Si bien es necesario profundizar en el análisis de contenidos, esta primera aproximación indica un gran involucramiento de las y los estudiantes y su solvencia para desarrollar actividades colaborativas enfocadas en su rol profesional, en un contexto de crisis.

Los resultados observados confirman las posibilidades de aplicación del aprendizaje colaborativo mediado como enfoque para evaluar actividades de enseñanza y aprendizaje complejas en entornos virtuales en el ámbito universitario.

\section{REFERENCIAS}

Bozkurt, A., Jung, I., Xiao, J., Vladimirschi, V., Schuwer, R., Egorov, G., Lambert, S., Al-Freih, M., Pete, J., Don Olcott, J., Rodes, V., Aranciaga, I., Bali, M., Alvarez, A. J., Roberts, J., Pazurek, A., Raffaghelli, J. E., Panagiotou, N., Coëtlogon, P. de, ... Paskevicius, M. (2020). A global outlook to the interruption of education due to COVID-19 pandemic: Navigating in a time of uncertainty and crisis. Asian Journal of Distance Education, 15(1), 1-126.

Cress, U., Stahl, G., Ludvigsen, S., \& Law, N. (2015). The core features of CSCL: Social situation, collaborative knowledge processes and their design. International Journal of Computer-Supported Collaborative Learning, 10(2), 109-116. https://doi.org/10.1007/s11412-0159214-2

Chávez, J., Barrera, R., Montaño, R., Sánchez, J., \& Fauré, J. (2021). Quality of Online Learning Participation in a Context of Crisis. Higher Learning Research Communications, 11. 10.18870/hlrc.v11i0.1203

Creswell, J., \& Plano, V. (2018). Designing and Conducting Mixed Methods Research. SAGE Publications Inc.

Czerwonogora, A. (2017). Análisis de foros colaborativos en línea en un curso de formación en TIC para docentes y profesionales de Enfermería. Virtualidad, Educación y Ciencia, 15 (8), 76-91. Recuperado de: https://revistas.unc.edu.ar/index.php/vesc/article/view/18 959

Edge, W. (2017). Nursing Professionalism: Impact of Social Media Use among Nursing Students. Journal of Healthcare Communications, 2(3). doi: 10.4172/24721654.100068

Garrison, D. R., \& Anderson, T. (2005). El e-learning en el siglo XXI. Barcelona: Octaedro.

Gibbs, G. (2012). El análisis de datos cualitativos en la investigación cualitativa. Madrid: Morata.

Gros Salvat, B. (2019). La investigación sobre el diseño participativo de entornos digitales de aprendizaje.
Recuperado

https://core.ac.uk/download/pdf/237483715.pdf

de:

Jackson, J., Fraser, R., \& Ash, P., (2014) Social Media and Nurses: Insights for Promoting Health for Individual and Professional Use. OJIN: The Online Journal of Issues in Nursing, 19(3). http://ojin.nursingworld.org/MainMenuCategories/ANA Marketplace/ANAPeriodicals/OJIN/TableofContents/Vo 1-19-2014/No3-Sept-2014/Insights-for-PromotingHealth.html

Kent, C., Laslo, E.,\& Rafaeli, S. (2016). Interactivity in online discussions and learning outcomes. Computers \& Education, $97, \quad 116-128$. https://doi.org/10.1016/j.compedu.2016.03.00

Lockhorst, D. (2004). Design principles for a CSCL environment in teacher training. IVLOS Series. Utrecht: IVLOS Institute of Education of Utrecht University.

Lockhorst, D., Admiraal, W., \& Pilot, A. (2010). CSCL in teacher training: what learning tasks lead to collaboration? Technology, Pedagogy and Education, 19(1), 63-78

Muñoz-Repiso, A. G.-V., Gómez-Pablos, V. B., \& García, C. L. (2014). Las TIC en el aprendizaje colaborativo en el aula de Primaria y Secundaria. Comunicar: Revista científica iberoamericana de comunicación y educación, $42,65-74$

Rodríguez Enríquez, C., y Barbieri, A. (2014). Construcción social del cuidado enfermero a partir de las redes sociales. Revista Uruguaya de Enfermería, 9(2). 30-37.

Rodríguez Enríquez, C. y Doninalli, M. (2021). Innovando en tiempos de Pandemia: estrategias de enseñanza enriquecidas con tecnologías dirigido a estudiantes de ciencias de la salud en redes sociales. Revista Uruguaya de Enfermeria, 16(2). https://doi.org/10.33517/rue2021v16n2a2

Schmitt, T. Sims-Giddens, S, y Booth, R. (2012). Social Media Use in Nursing Education. The Online Journal of Issues in Nursing, 17(3). Recuperado de: http://ojin.nursingworld.org/MainMenuCategories/ANA Marketplace/ANAPeriodicals/OJIN/TableofContents/Vo 1-17-2012/No3-Sept-2012/Social-Media-in-NursingEducation.html

Schrire, S. (2006). Knowledge building in asynchronous discussion groups: Going beyond quantitative analysis. Computers \& Education, 46(1), 49-70.

Scardamalia, M., \& Bereiter, C. (2006). Knowledge Building: Theory, Pedagogy, and Technology. En R. K. Sawyer (Ed.), The Cambridge Handbook of the Learning Sciences (1. ${ }^{\mathrm{a}}$ ed., pp. 97-116). Cambridge University Press. https://doi.org/10.1017/CBO9780511816833.008

Van Dijck, J. (2016). La cultura de la conectividad: una historia crítica de las redes sociales. Buenos Aires: Siglo XXI. 